\title{
Exponential stability of shallow water equations with arbitrary time dependent action
}

\author{
Ben Mansour Dia
}

Received: 14 October 2013 / Revised: 31 December 2013 / Accepted: 4 January 2014 / Published online: 21 January 2014

(C) Springer-Verlag Berlin Heidelberg 2014

\begin{abstract}
In this paper, we examine an exponential stability result in $L^{2}$-norm of the classical solution of shallow water equations. By using Riemann analysis with sub-critical flow condition, we show that only one boundary control law suffices to achieve the exponential stability. For that, we provide a sufficient dissipative condition as second order polynomial inequality with respect to the boundary control law which relies on the setting of a non-increasing arbitrary action.
\end{abstract}

Keywords Shallow water equations - Feedback control · Lyapunov stability $\cdot$ Riemann invariants

\section{Mathematics Subject Classification 35L50 - 76B75 .} 93D15 - 26A09

\section{Introduction}

It is well known that the stability of hydrodynamics processes plays an important role in engineering and physical sciences [1]. This is even more pronounced with the actual orientation of agricultural engineering in optimizing production, of physical engineering in monitoring flow in pipes to design supersonic engines. One of the key approaches that stabilize the dynamics is the boundary control. It follows that the methods of boundary control of flow play a key role in the development of the modern technology.

For finite dimensional dynamical systems, standard control procedure is to build a controller on models linearized around a set of different steady (or quasi-steady) "set-points".

\section{B. M. Dia (凶)}

SRI Center for Uncertainty Quantification in Computational Science and Engineering, CEMSE, King Abdullah University of Sciences and Technology, Thuwal 23955-6900, Kingdom of Saudi Arabia e-mail: mansourben2002@yahoo.fr; benmansour.dia@kaust.edu.sa
The control is then applied to the real nonlinear system by "gain scheduling" which means that the controller is dynamically chosen depending on the current state of the system. Most of the developed methods for controlling SW (shallow water) flow end up with control law exercised on the flow at the upstream and downstream boundaries. For example [2] presents an algebraic Faedo-Galerkin method of designing feedback control law so that the defined energy decreases exponentially. The work [3] stabilizes a section-flow formulation of the one dimensional SW equations around a constant state using LQ (linear quadratic) technique developed in [4]. Due to the Riemann invariants performance of tracking propagating information, several methods based on the Lyapunov stability have been developed [5-8]. In [5], explicit sufficient dissipative condition is given and applied to a sloping channel flow. A similar approach is used in [6] to regulate the water flow by an appropriate choice of the boundary conditions. This latter method uses only measurements of the state at the boundaries and it is improved in [9] by including the so-called integral action which achieves local convergence of the state.

However, all the above works used upstream and downstream controllers to achieve exponential stability. Implementation of those methods requires upstream and downstream control plants. Now, putting the study of flow regulation on the framework of cost is a major advance to state on the profitability and optimality. In this paper, we aim to stabilize the entire system with cheap control in the sense that its implementation needs only upstream control plant. We address a "one gate" control problem for the 1-D SW equations. The control problem is subject to the initial condition and a wall condition for the perturbation state at the downstream boundary. The control problem consists in stabilizing the hydrodynamical variables (height and flow) around an equilibrium set named steady-state which is supposed known 
in advance. Based on Riemann invariants analysis, we propose a new technique of designing feedback controller as boundary condition for the flow. The innovative light in our proposed method is that

(i) the control law uses only the upstream boundary value of the solution and the initial condition,

(ii) the design of the control law relies on an arbitrary timedelay function called action.

This paper is organized as follows. In Sect. 2, we present the 1-D SW model, linearize it around the equilibrium set and formulate the stabilization problem. Section 3 is devoted to formal computations to write out the Riemann invariants governing the perturbation waves and also to delineate the mathematical framework of the control problem. In Sect. 4, we present the main result and its proof. Section 5 states numerical details and experiments to assess the effectiveness of the control law.

\section{Shallow water equations}

It is widely accepted that the unsteady flow of water in a two-dimensional space may be described by the SW equations, which are mass and momentum conservation laws [10] and can be obtained by depth averaging the Navier-Stokes equations in the vertical direction. By neglecting diffusion of momentum due to turbulence, this leads to a 2-D formulation in terms of depth averaged quantities and the water depth itself, see [11]. If one considers further a physical domain whose one horizontal dimension is much greater than the second one, the flow obeys to the following 1-D hyperbolic conservative law

$\partial_{t} U+\partial_{x} F(U)=0$,

where $U$ is the hydrodynamical variable vector and $F(U)$ the flux functional

$U=\left(\begin{array}{c}h \\ h u\end{array}\right)$ and $F(U)=\left(\begin{array}{c}h u \\ h u^{2}+\frac{1}{2} g h^{2}\end{array}\right)$,

where the constant $g$ is the gravitational force acting on the fluid. The variable $h$ represents the height of the water column while $u$ is the velocity along the channel. The notations $\partial_{t}$ and $\partial_{x}$ stand for the derivative with respect to the time $t$ and the space variable $x$ respectively. The system (1) is also called Saint-Venant equations [12]. For a horizontal prismatic canal with rectangular flow section and unit width, we introduce the flow variable $q=h u$, the hyperbolic system (1) can be recast in the following detailed form

$$
\begin{array}{ll}
\partial_{t} h+\partial_{x} q=0 & \text { in } \mathcal{Q}, \\
\partial_{t} q+g h \partial_{x} h+\partial_{x}\left(\frac{q^{2}}{h}\right)=0 & \text { in } \mathcal{Q}
\end{array}
$$

where $(t, x) \in \mathcal{Q}=(0, T) \times\left[L_{1}, L_{2}\right], T$ is a positive real number which is assumed to be enough large.

Let $(\bar{h}, \bar{q})$ be a steady state which is the target state of the stabilization process. This steady state $(\bar{h}, \bar{q})$ is solution of the stationary problem associated to (2). The control purpose is to design upstream boundary condition so that the flow variable vector $(h, q)$ is exponentially stable around $(\bar{h}, \bar{q})$. In other terms, the control problem is the stabilization problem of the perturbation state $(h, q)-(\bar{h}, \bar{q})$ around $(0,0)$.

\subsection{Linearization}

We derive here the model governing the evolution of the perturbation state denoted by $(\tilde{h}, \tilde{q})$. This residual state is introduced as the difference between the whole state $(h, q)$ and the steady state $(\bar{h}, \bar{q})$

$$
(\tilde{h}, \tilde{q})(t, x)=(h, q)(t, x)-(\bar{h}, \bar{q}) \text {. }
$$

We use the assumptions $|\tilde{h}| \ll \bar{h}$ and $|\tilde{q}| \ll|\bar{q}|$ when plugging (3) into (2). Since the control problem is addressed for a sub-critical flow regime, we can neglect the nonlinear perturbation terms to write the following linearized model:

$\partial_{t} \tilde{U}+A \partial_{x} \tilde{U}=0$ in $\mathcal{Q}$,

where $\tilde{U}$ is the residual state vector and $A$ is the flux matrix

$\tilde{U}=\left(\begin{array}{c}\tilde{h} \\ \tilde{q}\end{array}\right)$ and $A=\left(\begin{array}{cc}0 & 1 \\ c^{2}-\bar{u}^{2} & 2 \bar{u}\end{array}\right)$,

where $\bar{u}=\bar{q} / \bar{h}$ and the quantity $c=\sqrt{g \bar{h}}$ is the wave speed. Next, we formulate the feedback control problem associated to the linearized system (4).

\subsection{Boundary feedback control problem}

We consider the following stabilization problem by boundary control

$$
\begin{cases}\partial_{t} \tilde{h}+\partial_{x} \tilde{q}=0 & \text { in } \mathcal{Q}, \\ \partial_{t} \tilde{q}+\left(c^{2}-\bar{u}^{2}\right) \partial_{x} \tilde{h}+2 \bar{u} \partial_{x} \tilde{q}=0 & \text { in } \mathcal{Q}, \\ \tilde{h}(0, x)=\tilde{h}^{0}(x), \quad \tilde{q}(0, x)=\tilde{q}^{0}(x) & \text { in } \Omega, \\ \tilde{q}\left(t, L_{2}\right)=0 & \text { on }(0, T), \\ \tilde{q}\left(t, L_{1}\right)=\tilde{q}_{L_{1}}(t) & \text { on }(0, T) .\end{cases}
$$

The time dependent function $\tilde{q}_{L_{1}}(t)=0$ is the stabilizing control law for the upstream volumetric flow while $\tilde{q}\left(t, L_{2}\right)$ arises from the wall condition $\tilde{u}\left(t, L_{2}\right)=0$. In opposite to the classical methods, the regulation process operates only with one law instead of two. At the upstream boundary the controller $\tilde{q}_{L_{1}}(t)$ will be defined by means of an arbitrary non-increasing action. A reflecting boundary condition is set at downstream $L_{2}$ as depicted in Fig. 1. 


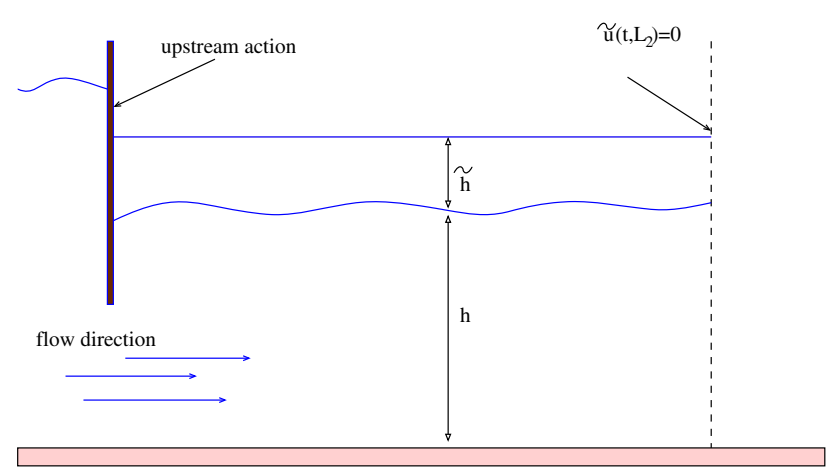

Fig. 1 Domain $\Omega$ treatment

\section{Computational analysis}

In this section, the computations showcase the eigenstructure of the linear hyperbolic system (4) and the variational formulation is presented. Those will be used for the controller building process.

\subsection{Characteristic variables}

Let $\lambda_{1}$ and $\lambda_{2}$ be the eigenvalues of the matrix $A$

$\lambda_{1}=\bar{u}-c \quad$ and $\quad \lambda_{2}=\bar{u}+c$.

The transformation matrix $P$ of the eigenvectors of $A$ is written as

$P=\left(\begin{array}{cc}1 & 1 \\ \lambda_{1} & \lambda_{2}\end{array}\right)$

The diagonal matrix of the eigenvalues is denoted by $\Lambda=$ $\operatorname{diag}\left\{\lambda_{1}, \lambda_{2}\right\}$. Then the hyperbolic linear system follows

$\partial_{t} \tilde{U}+P \Lambda P^{-1} \partial_{x} \tilde{U}=0$

Multiplying left-hand side of the system (6) by the matrix $P^{-1}$ yields

$\partial_{t}\left(P^{-1} \tilde{U}\right)+\Lambda \partial_{x}\left(P^{-1} \tilde{U}\right)=0$.

In this sequel, we introduce the vector of characteristic variables $\xi=P^{-1} \tilde{U}=\left(\xi_{1}, \xi_{2}\right)^{t r}$ where

$\xi_{1}(t, x)=\frac{1}{2 c}\left(\lambda_{2} \tilde{h}(t, x)-\tilde{q}(t, x)\right)$,

$\xi_{2}(t, x)=\frac{1}{2 c}\left(-\lambda_{1} \tilde{h}(t, x)+\tilde{q}(t, x)\right)$.

The variables $\xi_{1}$ and $\xi_{2}$ are gravity waves and specify the boundary conditions.

Remark 1 The steady flow is supposed sub-critical. This assumption implies that $\lambda_{1}<0<\lambda_{2}$ which means that $\xi_{1}$ describes the outgoing wave at $L_{1}$ while $\xi_{2}$ represents the incoming wave at $L_{1}$.

\subsection{Variational formulation}

We now describe the variational formulation of the linearized control problem (5). Recall that $\mathcal{Q}=(0, T) \times \Omega$, we define the functional space $H(\operatorname{Div}, \mathcal{Q})$ as

$H(\operatorname{Div}, \mathcal{Q})=\left\{\Psi \in L^{2}(\mathcal{Q})^{2} ; \quad \operatorname{Div}(\Psi) \in L^{2}(\mathcal{Q})\right\}$,

where the differential operator Div is given by

$\operatorname{Div} \Psi=\partial_{t} \Psi_{1}+\partial_{x} \Psi_{2}$

If the vector $(\tilde{h}, \tilde{q})$ satisfies (5) and belongs to $L^{2}(\mathcal{Q})^{2}$, then for $i=1,2$ the vector $X_{i} \in H(\operatorname{Div}, \mathcal{Q})$ since $\operatorname{Div}\left(X_{i}\right)=$ $0 \in L^{2}(\mathcal{Q})$ where

$X_{1}(\tilde{h}, \tilde{q})=\left(\begin{array}{l}\tilde{h} \\ \tilde{q}\end{array}\right), X_{2}(\tilde{h}, \tilde{q})=\left(\begin{array}{c}\tilde{q} \\ \left(c^{2}-\bar{u}^{2}\right) \tilde{h}+2 \bar{u} \tilde{q}\end{array}\right)$.

In this sense, we define the functional space $V$ by

$V=\left\{\begin{array}{c}(\tilde{h}, \tilde{q}) \in L^{\infty}\left(0, T ; L^{2}(\Omega)^{2}\right): \text { for } i=1,2 \\ X_{i}(\tilde{h}, \tilde{q}) \in H(\operatorname{Div}, \mathcal{Q}), \quad \tilde{q}\left(\cdot, L_{2}\right)=0\end{array}\right\}$.

The trace of the solution $(\tilde{h}, \tilde{q})$ belonging to $V$ makes sense in $H^{-\frac{1}{2}}(\partial \mathcal{Q})$ which is the dual of $H^{1}(\mathcal{Q})$ (see [13], p. 27). In this setting, we consider the following weak form

For all $(\psi, \varphi) \in V$, find $(\tilde{h}, \tilde{q})$ in $V$ such that :
$\int_{L_{1}}^{L_{2}} \psi \partial_{t} \tilde{h} d x-\int_{L_{1}}^{L_{2}} \tilde{q} \partial_{x} \psi d x=\psi\left(L_{1}, t\right) \tilde{q}_{L_{1}}(t)$,
$\int_{L_{1}}^{L_{2}} \varphi \partial_{t} \tilde{q} d x-\left(c^{2}-\bar{u}^{2}\right) \int_{L_{1}}^{L_{2}} \varphi \partial_{x} \tilde{h} d x$
$-2 \bar{u} \int_{L_{1}}^{L_{2}} \tilde{q} \varphi d x=2 \bar{u} \varphi\left(t, L_{1}\right) \tilde{q}_{L_{1}}(t)$.

In the rest of this paper, the variational formulation of (5) appeals to (8). In the next section, we define the quantity to be stabilized and we state the main result.

\section{Exponential stability analysis}

Definition 1 We consider the following energy of (5):

$E(t)=\sum_{i=1}^{2} \int_{L_{1}}^{L_{2}} \xi_{i}^{2}(t, x) \exp \left(-\frac{\mu}{\lambda_{i}} x\right) d x$,

where $\mu$ is an arbitrary positive number and stands for the stabilization rate.

The quantity $E(t)$ is to be minimized. The stabilization rate $\mu$ quantifies the speed of the energy decay in the sense that large $\mu$ provide faster energy decay. As we will see below, the quantity $E(t)$ is a Lyapunov function candidate and it measures the perturbation state $(\tilde{h}, \tilde{q})$ over the whole 
domain $\left[L_{1}, L_{2}\right]$. The objective is to design a boundary condition at $L_{1}$ such that $E(t)$ decreases towards 0 . In order to stabilize $E(t)$, we first estimate it in the next subsection.

\subsection{Energy estimation}

For $i=1,2$ we integrate over $\left[L_{1}, L_{2}\right]$ the product of $\frac{\partial \xi_{i}}{\partial t}(t, x)+\lambda_{i} \frac{\partial \xi_{i}}{\partial x}(t, x)$ by $\xi_{i}(t, x) \exp \left(-\frac{\mu}{\lambda_{i}} x\right)$ and sums the two obtained relations to write the following energy estimation

$$
\frac{d E(t)}{d t}+\mu E(t)=-\sum_{i=1}^{2} \lambda_{i}\left[\xi_{i}^{2}(t, x) \exp \left(-\frac{\mu}{\lambda_{i}} x\right)\right]_{L_{1}}^{L_{2}} .
$$

From the estimation (10), to obtain an exponential decay of the energy $E(t)$ in time, the RHS of (10) should be nonpositive.

\subsection{Main result}

We now present our main result.

Theorem 1 Let $T_{0}=\left(L_{2}-L_{1}\right) / \lambda_{2}$, if the initial condition $\tilde{U}^{0} \in L^{2}(\Omega)^{2}$, then there exists a boundary controller $\tilde{q}_{L_{1}}(t)$ at $L_{1}$ such that the control system (5) has a unique solution $\tilde{U} \in V$ which is exponentially stable around $(0,0)^{t r}$ in the sense that

$E(t) \leq E\left(T_{0}\right) \exp (-\mu t)$ for $t>T_{0}$.

It is shown in [14] that the linearized SW system admits a unique solution when the data (initial and boundary conditions) are smooth and $T$ is bounded. However, in a stabilization problem as it is case here, the time domain is not supposed to be bounded. So we demonstrate the existence of solution using Faedo-Galerkin method different to the method in [14]. The controller $\tilde{q}_{L_{1}}(t)$ is designed by means of a boundary action which is arbitrary in a certain time-interval. Stabilizing boundary controller for hyperbolic conservation law is designed according to dissipative boundary condition [8].

Proof For sake of clarity, we proceed by steps.

Existence of solution Replacing the test function $(\psi, \varphi)$ by

$\tilde{U}^{t r}\left(P^{-1}\right)^{t r} \exp \left(-\mu x \Lambda^{-1}\right) P^{-1}$

in the weak form (8), the energy estimate (10) yields. Following the procedure developed in [11], the existence of weak solution follows.

Definition of the action Let $L=L_{2}-L_{1}$ be the length of the hydraulic canal, from the energy estimate (10), we state the following dissipative boundary conditions $\xi_{1}^{2}\left(t, L_{1}\right)-\xi_{1}^{2}\left(t, L_{2}\right) \exp \left(-\mu \frac{L}{\lambda_{1}}\right) \leq 0$,

$\xi_{2}^{2}\left(t, L_{1}\right)-\xi_{2}^{2}\left(t, L_{2}\right) \exp \left(-\mu \frac{L}{\lambda_{2}}\right)=0$.

It is worth noticing that (12)-(13) are sufficient exponential stability conditions for (5). We introduce then the boundary action as a damping function acting on the incoming wave at the upstream boundary $L_{1}$. The idea is to define the upstream action $\alpha$ using (13) and then determine the corresponding flow rate $\tilde{q}_{L_{1}}(t)$ satisfying (12).

To express the boundary action $\alpha$, we consider the second characteristic equation

$\frac{\partial \xi_{2}}{\partial t}(t, x)+\lambda_{2} \frac{\partial \xi_{2}}{\partial x}(t, x)=0$.

Let $T_{0}=\frac{L}{\lambda_{2}}$, we integrate (14) over the characteristic line from $\left(t, L_{1}\right)$ to $\left(t+T_{0}, L_{2}\right)$ to get

$\xi_{2}\left(t, L_{1}\right)=\xi_{2}\left(t+T_{0}, L_{2}\right)$.

Then we act on the $L_{1}$-incoming characteristic variable $\xi_{2}$ at the boundary $L_{1}$ :

$\xi_{2}\left(t, L_{1}\right)=\alpha(t)$

where $\alpha$ is a time dependent function. Therefore, using (15) we get for $t \geq T_{0}$

$\xi_{2}\left(t, L_{2}\right)=\xi_{2}\left(t-T_{0}, L_{1}\right)=\alpha\left(t-T_{0}\right)$.

We can rewrite then (13) as

$\alpha^{2}(t)-\alpha^{2}\left(t-T_{0}\right) \exp \left(-\mu T_{0}\right)=0$.

Finally, to satisfy (13), we have to define the action function $\alpha$ such that:

$\left\{\begin{array}{l}\alpha(0)=\xi_{2}^{0}\left(L_{1}\right), \\ \alpha(t)=\alpha\left(t-T_{0}\right) \exp \left(-\mu \frac{T_{0}}{2}\right) \forall t \geq T_{0} .\end{array}\right.$

Boundary controller at $L_{1}$ The flow rate at the upstream boundary is completely defined by the prescription of the controller $\tilde{q}_{L_{1}}(t)$ corresponding to the control action $\alpha$ defined at (18). For that, we write (12) as second order inequality with respect to $\tilde{q}_{L_{1}}(t)$. The height variable at the boundaries $\left(\tilde{h}\left(t, L_{1}\right)\right.$ and $\left.\tilde{h}\left(t, L_{2}\right)\right)$ are expressed using the condition $\tilde{q}\left(t, L_{2}\right)=0$ and the action definition. For $t \geq T_{0}$

$\tilde{h}\left(t, L_{1}\right)=\frac{1}{\lambda_{1}}\left(\tilde{q}_{L_{1}}(t)-2 c \alpha(t)\right)$,

and

$\tilde{h}\left(t, L_{2}\right)=\frac{2 c}{-\lambda_{1}} \alpha\left(t-T_{0}\right)$.

Plugging the above relations in the expressions of the Riemann invariant $\xi_{1}$, it comes

$\xi_{1}\left(t, L_{1}\right)=\frac{1}{\lambda_{1}}\left(\tilde{q}_{L_{1}}(t)-\lambda_{2} \alpha(t)\right)$ 
and

$\xi_{1}\left(t, L_{2}\right)=\frac{\lambda_{2}}{-\lambda_{1}} \alpha\left(t-T_{0}\right)$.

Using (19) and (20), the dissipative condition (12) is recasting in the following form

$$
\begin{gathered}
\frac{1}{\lambda_{1}} \tilde{q}_{L_{1}}^{2}(t)-2 \frac{\lambda_{2}}{\lambda_{1}^{2}} \alpha(t) \tilde{q}_{L_{1}}(t)+\left(\frac{\lambda_{2}}{-\lambda_{1}}\right)^{2} \alpha^{2}(t) \\
-\left(\frac{\lambda_{2}}{\lambda_{1}}\right)^{2} \alpha^{2}\left(t-T_{0}\right) \exp \left(-\frac{\mu}{\lambda_{1}}\right) \leq 0 .
\end{gathered}
$$

Reminding that $\alpha$ satisfies (18) and the eigenvalue $\lambda_{1}$ is negative, we write (21) as follows

$\tilde{q}_{L_{1}}^{2}(t)-2 \frac{\lambda_{2}}{\lambda_{1}} \alpha(t) \tilde{q}_{L_{1}}(t) \geq 0$.

This last relation is satisfied by the following general setting of the controller

$\tilde{q}_{L_{1}}(t)=2 \frac{\lambda_{2}}{\lambda_{1}}(\theta \min (\alpha(t), 0)+(1-\theta) \max (\alpha(t), 0))$,

where $\theta \in[0,1]$. However the boundary control law is seeking with the non-nullity constraint $\left(\tilde{q}_{L_{1}}(t) \neq 0\right)$ for $T_{0}<t<T_{0}+\varepsilon, \varepsilon>0$. In view of the properties of the Lyapunov function candidate $E(t)$, we choose

$\tilde{q}_{L_{1}}(t)=2 \frac{\lambda_{2}}{\lambda_{1}} \alpha(t)$ for $t \geq T_{0}$

The above relation is the controller and represents the boundary condition at $L_{1}$ for $t \geq T_{0}$. The boundary condition at $L_{1}$ over the time interval $\left[0, T_{0}\right]$ is an arbitrary function. It is arbitrary in the sense that any non-increasing function in the time interval $\left[0, T_{0}\right]$ suffices.

Finally we have shown the exponential decay. This is provided by the non-positivity of $\frac{d E(t)}{d t}+\mu E(t)$ for $t \geq T_{0}$ which is guaranteed by the setting of the boundary control law (24). The proof is then complete.

Let remark some properties of the boundary controller related to the action and the problem data.

Remark 2 For $t \geq T_{0}$, the action is completely defined and variates according to

$\alpha(t)=\alpha\left(t-T_{0}\right) \exp \left(-\frac{\mu T_{0}}{2}\right) ;$

the action is then a time-delay function. Therefore the boundary flow $\tilde{q}_{L_{1}}(t)$ is a time-delay feedback controller.

Remark 3 According to the initial conditions, we define the initial total volumetric flow of perturbation $V_{0}$ as

$V_{0}=\int_{L_{1}}^{L_{2}}\left(c \tilde{h}^{0}(x)+\tilde{q}^{0}(x)\right) d x$. (i) If $V_{0}<0$ the control task is to fulling the canal, the steady velocity satisfies $0<\bar{u}<c$.

(ii) If $V_{0}>0$ the stabilization is achieved by reducing the water level to $\bar{h}$. In that case the steady velocity satisfies $-c<\bar{u}<0$.

\section{Numerical experiments}

This section is devoted to numerical examples to illustrate how the controller acts on the flow. We will use several type of boundary actions to highlight the arbitrariness of the action $\alpha$ on the time interval $\left[0, T_{0}\right]$. We start by evaluating the performance of the proposed controller on the linearized SW equations. Then, we state and analyze the control problem for the nonlinear SW system.

\subsection{Numerical simulations of the linearized model}

The first order upwind method (see [15]) is applied to system (5). The space domain $[0,10]$ is meshed with $d x=0.2$ and the numerical experiment consists in vanishing the following initial condition

$\left\{\begin{array}{l}\tilde{h}^{0}(x)=0.5+0.075 * \sin (2 \pi x), \\ \tilde{q}^{0}(x)=0 .\end{array}\right.$

The steady state parameters are $\bar{h}=1 \mathrm{~m}$ and $\bar{u}=$ $-0.25 \mathrm{~m} \mathrm{~s}^{-1}$. According to the problem data, the initial total volumetric flow $V_{0}$ is positive. Therefore the control task is achieved by the outflow $\tilde{q}_{L_{1}}(t)$. Several experiments with respect to the action definition are done in order to showcase the arbitrariness of the boundary action. From the problem data, the time-constant is $T_{0}=3.469 \mathrm{~s}$, we consider then the following action definitions for $t \in\left[0, T_{0}\right]$;

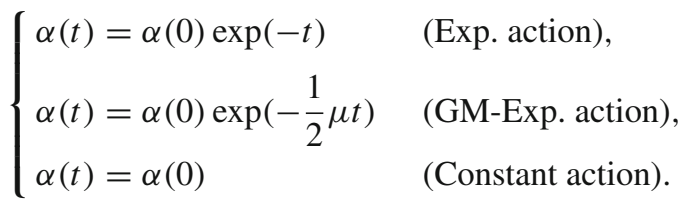

The Fig. 2 describes the variation of the boundary controller $\tilde{q}_{L_{1}}(t)$ according to the above actions. The nonmonotonicity in the general setting (18) of the action $\alpha$ appears for the exponential action (Exp. action).

With the same initial conditions (25), we observe the variation of the energies corresponding to the above actions in the following figure.

As expected from the theoretical result, for any nonincreasing action in the time interval $\left[0, T_{0}\right]$, the boundary controller $\tilde{q}_{L_{1}}(t)$ guarantees an exponential decay of the energy $E$. The decay behavior depends on the type of the boundary action. The exponential action provides the faster decay. Generally, the monotonicity of the energy decay is not 


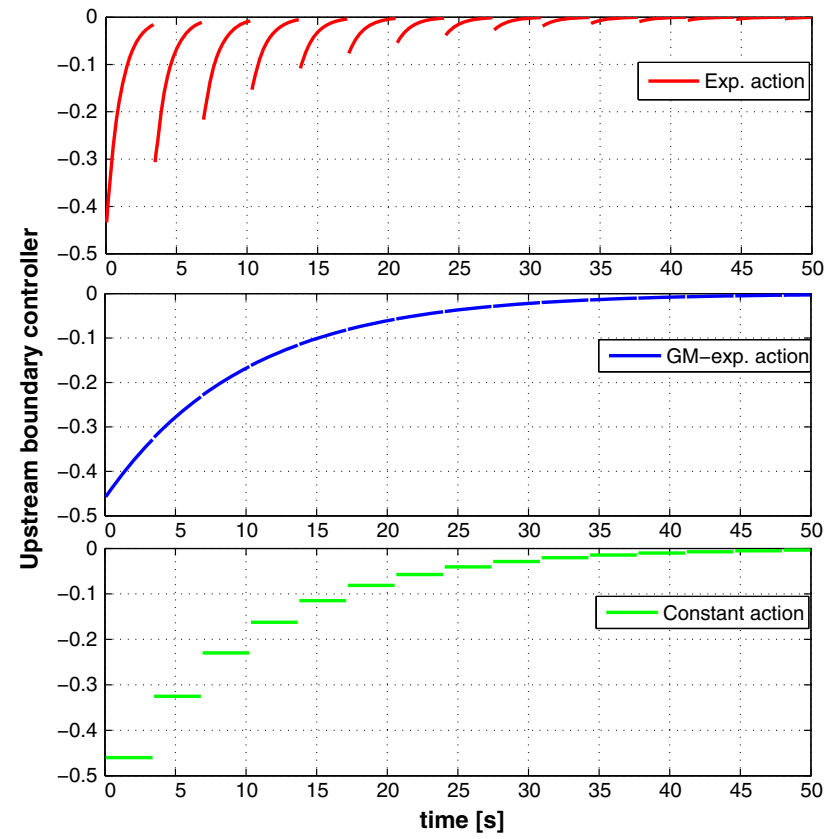

Fig. 2 Upstream boundary controller $\tilde{q}_{L_{1}}(t)$ according to some actions: Exponential action (Exp. action) at the top, Global monotone exponential action (GM-Exp. action) at the middle and constant action (Constant action) at the bottom

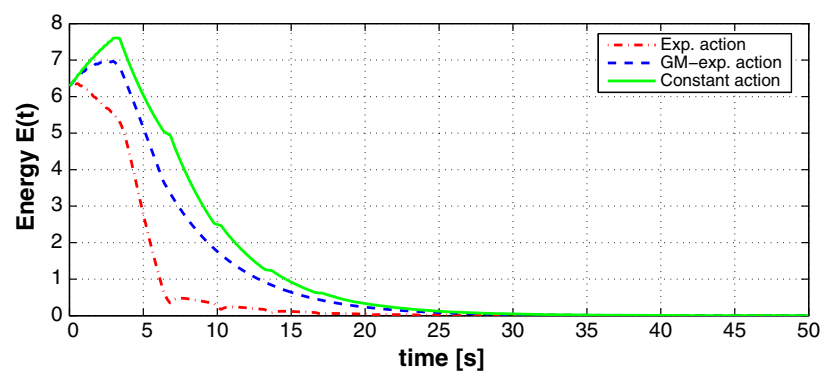

Fig. 3 Behavior of the energy variation according to some actions: Exp. action, GM-Exp. action and Constant action

expected due to the discontinuity of the boundary action at $x=k T_{0}$ for $k \in \mathbb{N}^{*}$. this is the case for constant action and exponential action. However a decreasing monotone (global non-increasing monotone) action provides a monotone convergence of the energy. It is illustrated in Fig. 3 where the energy associated to the GM-exponential action has a monotone decay.

\subsection{Control of nonlinear shallow water equations}

In this part, the boundary control law (24) is accommodated to the nonlinear SW equations and numerical experiments are performed. The problem is tackled numerically through the following system

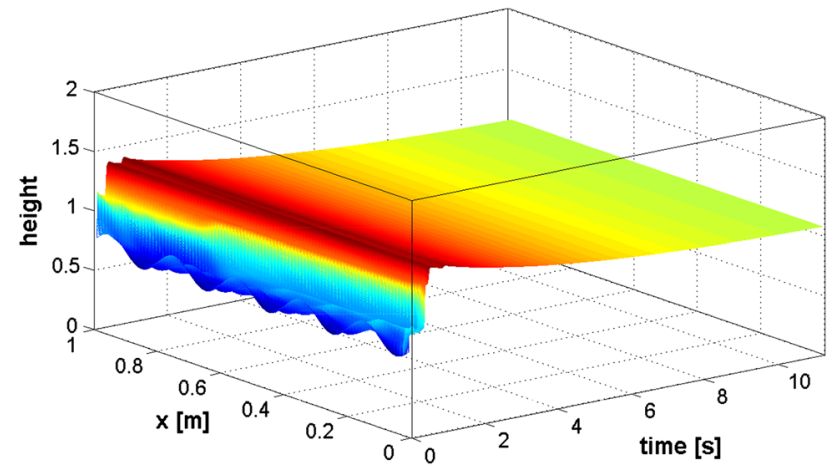

Fig. 4 Stabilization dynamics of the height of the controlled nonlinear waves

$\begin{cases}\partial_{t} h+\partial_{x} q=0 & \text { in } \mathcal{Q}, \\ \partial_{t} q+g h \partial_{x} h+\partial_{x}\left(\frac{q^{2}}{h}\right)=0 & \text { in } \mathcal{Q}, \\ h(0, x)=h^{0}(x), q(0, x)=q^{0}(x) & \text { for } x \in\left[L_{1}, L_{2}\right], \\ q\left(t, L_{1}\right)=\bar{h} \bar{u}+\tilde{q}_{L_{1}}(t) & \text { for } t>0, \\ q\left(t, L_{2}\right)=\bar{h} \bar{u} & \text { for } t>0 .\end{cases}$

A second order finite volumes scheme with Roe flux is applied to (27). The stability of the scheme runs under the CFL condition. We are interested in observing the dynamics of the controlled nonlinear waves. For that, we consider the following state as initial conditions

$\left\{\begin{array}{l}h^{0}(x)=\bar{h}-(0.25+0.1 * \sin (10 \pi x)), \\ q^{0}(x)=\bar{h} \bar{u} .\end{array}\right.$

With a global non-increasing action (GM-exp. action) defined at (26), the boundary controller $\tilde{q}_{L_{1}}(t)$ achieves the stabilization of the water level around $\bar{h}=1 \mathrm{~m}$ with constant velocity $\bar{u}=0.25 \mathrm{~m} \mathrm{~s}^{-1}$. For the stabilization rate $\mu=0.5$ and the stability condition $C F L=0.2$. The behavior of the controlled nonlinear waves in the space domain $[0,1]$ is displayed in Fig. 4.

Finally we orientate our interest in the behavior of the energy variation according to the stabilization rate. For that, we simulate an example where the control task consists in fulling-up a canal $[0,5]$ from the static initial state $h^{0}(x)=$ $\bar{h}-0.25$ to the state $\bar{h}=1 \mathrm{~m}$ with the constant velocity $\bar{u}=0.15 \mathrm{~m} \mathrm{~s}^{-1}$.

The perturbation energy computed from the nonlinear problem is plotted in Fig. 5. Using an exponential action (Exp. action) at (26), we showcase the influence of the stabilization rate $\mu$ on the convergence rate. Higher stabilization rate provides faster convergence. 


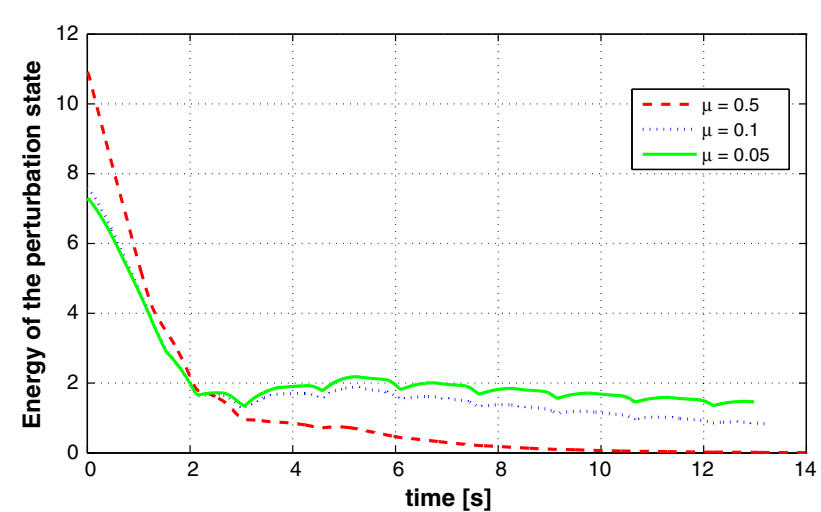

Fig. 5 Variation of the energy computed from the nonlinear control problem according the stabilization rate

\section{Conclusion}

In this paper, we have proposed a one-gate control method to stabilize hydraulic 1-D flow modeled by SW equations. The technique uses an arbitrary time dependent function as upstream action while wall condition for the waves of the perturbation state is set to downstream boundary. The proposed control law provides exponential convergence of the state toward the steady state. Many numerical simulations with different values of stabilization rate, different initial conditions and more importantly different types of actions have been presented. In future, we aim to adapt this approach to control 2-D hydraulic flow.

\section{References}

1. Swaters GE (1999) Introduction to hamiltonian fluid dynamcs and stability theory, 1st edn. chapman and Hall/CRC

2. Sene A, Wane AB, Le Roux D (2008) Control of irrigation channels with variable bathymetry and time dependent stabilization rate. C R Acad Sci Paris Ser 1346:1119-1122

3. Chen ML, Georges D, Lefevre L (2002) Infinite dimensional LQ control of an open channel hydraulic system, The 4th Asian control conference Sept 25-26, Singapore

4. Russell DL (1987), Controllability and stabilizability theory for linear partial differential equations: Recent progress and opens questions. SIAM Rev 20(4)
5. Bastin G, Coron JM, d'Andréa-Novel B (2009) On Lyapunov stability of linearised Saint-Venant equations for a sloping channel. Netw Heterog Media 4(2):177-187

6. Coron JM, D'Andrea Novel B (2007) A strict lyapunov function for boundary control of hyperbolic systems of conservation laws. IEEE Trans Autom Control 52(1):2-11

7. Coron JM, D'Andrea Novel B, Bastin G (1999) A lyapunov approach to control irrigation canals modeled by Saint Venant equations, CD-Rom Proceedings, ECC 99, Karlsruhe, Germany

8. de Halleux J (2004) Boundary control of quasi-linear hyperbolic Initial boundary value problem. Université Catholique de Louvain, Thése de doctorat

9. Dos Santos V, Bastin G, Coron JM, d Andréa-Novel B (2008) Boundary control with integral action for hyperbolic systems of conservations laws: stability and experiments. Automatica 44(5):1310-1318

10. Marche F (2008) Derivation of a new two-dimensional viscous shallow water model with varying topography, bottom friction and capillary effects. Eur J Mech B/Fluids 26:49-63

11. Dia BM, Opplestrup J (2013) Stability by boundary control of 2-D shallow water equations. Int J Dynam Control 1(1):41-53

12. Saint-Venant AJC (1871) Théorie du mouvement non permanent des eaux avec applications aux crues des riviéres et á l'introduction des marées dans leur lit, comptes rendus de 1Académie des Sciences de Paris 73:148-154 et 237-240

13. Girault V, Riviart PA (1986) Finite elements methods for navier stokes equations, theory and algorithms, vol 136 of mathematical surveys and monographs. Springer, Providence

14. Besson O, Kane S, Sy M (2007) On a 1D-Shallow water model: existence of solution and numerical simulations. Revue Arima 9:247-260

15. Leveque RJ (2002) Finite volume methods for hyperbolic problems. Cambridge Texts in Applied Mathematics, Cambridge

16. Batlle V, Pérez RR, Rodriguez LS (2007) Fractional robust control of main irrigation canals with variable dynamic parameters. Control Eng Pract 15(6):673-686

17. Leveque RJ (1997) Wave propagation algorithms for multidimensional hyperbolic systems. J comput Phys 131:253-327

18. N'diaye M, Bastin G (2004) Command frontiére d'un bief de canal avec prelévements inconnus. Journal Européen des Systémes Automatisés 38(3-4):347-372

19. Pelanti M, Bouchut F. Mangeney A (2008) A Roe-Type scheme for two-phase shallow granular flows over variable topography, M2AN, pp 851-885

20. Weyer E (2003) LQ control of irrigation channel. Decis Control $1: 750-755$ 